\title{
Die „Gesundheitsgesellschaft" - eine Herausforderung für eine theoretisch orientierte Gesundheits- und Medizinsoziologie? Eine Einleitung
}

\author{
Karl Krajic $\cdot$ Rudolf Forster $\cdot$ Elisabeth Mixa
}

\section{Warum gerade jetzt ein Schwerpunktheft zum Thema „Gesundheit“ in einer allgemein- soziologischen Zeitschrift?}

Konkreter Anlass ist eine theoretische Diskussion, die die Sektionen für Gesundheitsund Medizinsoziologie Deutschlands, Österreichs und der Schweiz im Rahmen einer Konferenz im steirischen Bad Gleichenberg im März 2008 initiiert hatten (siehe dazu den Tagungsbericht im Heft 3/08 der ÖZS). Sie zentrierte um ein Buch, das die Autorin Ilona Kickbusch „Die Gesundheitsgesellschaft“ getitelt hatte (Kickbusch 2006). Die Organisatorinnen und Organisatoren der Konferenz waren der Meinung, dass sich die Gesundheits- und Medizinsoziologie pro-aktiv in dieser Diskussion verhalten sollte, und das systematisch und unter Anschluss an soziologische Theorie. Das heißt in Bezug auf Kickbusch: die Thesen ernst nehmen, in ihrem Inhalt und auch als Anstoß, selbst etwas grundsätzlicher und theoretischer über die gesellschaftliche Einbettung ihres Gegenstands in gesellschaftliche Evolution nachzudenken - und hier besonders mit Fokus auf die Bedeutungsveränderung bzw. -zunahme von Gesundheit in unserer Gesellschaft. Angesichts einer in den letzten Jahrzehnten oft kritisierten, eher empiristischen bzw. „anwendungslastigen“ Orientierung der Gesundheits- und Medizinsoziologie war das kein selbstverständliches Unternehmen. Wie gut es geglückt ist, kann die Leserin/der Leser nunmehr selbst beurteilen. Das vorliegende Heft versammelt drei zentrale Theorieinputs der Konferenz als Artikel sowie einige in unterschiedlicher Weise exemplarische Forschungsnotizen.

\section{Zur Bedeutung von Medizin und Gesundheit für die Soziologie}

Als Einleitung und vor allem an jene Leser/innen gerichtet, denen das Feld Gesundheitsund Medizinsoziologie und seine Traditionen nicht so nahe sind, beginnen wir mit einigen kurzen Hinweisen auf wichtige Meilensteine der Beziehungen zwischen der Subdisziplin und der „Mutterdisziplin“ Soziologie.

Historisch haben Fragen des Einflusses der gesellschaftlichen Verhältnisse auf Lebenserwartung, auf Krankheit und Gesundheit der Menschen bzw. Fragen des gesellschaftlichen Umgangs mit Gesundheit/Krankheit die Soziologie durchaus interessiert. Die Aufmerksamkeit Emile Durkheims für den Selbstmord, der hohe Stellenwert, den 
die Arzt-Patienten-Beziehung als Beispiel in der Gesellschaftsanalyse von Talcott Parsons hatte, auch das spezifische Interesse von Erving Goffman, Anselm Strauss (und anderen „Interaktionisten“) für medizinisch-pflegerische Organisationen bzw. Institutionen seien hier exemplarisch genannt. Zudem spielte auch in der Parsons-Kritik die Medizin als gesellschaftlicher Bereich eine wichtige Rolle, z. B. in Eliot Freidsons „Dominanz der Experten“".

Auch in der gegenwärtigen Soziologie dürfte es weitgehend konsensfähig sein, dass Themen rund um Medizin, Krankheit, aber auch Gesundheit in unserer Gesellschaft aktuell eine hohe Aufmerksamkeit und Wertschätzung genießen. Nicht mehr ganz so konsensfähig ist wohl die Behauptung einer massiven relativen Bedeutungszunahme gesellschaftlicher Kommunikation über Krankheit und Gesundheit in den letzten fünfzig Jahren. Es gibt jedenfalls eine Reihe von Indikatoren dafür, dass sich ein spezialisiertes, komplexes, dynamisches System der Medizin/Krankenversorgung ausdifferenziert hat, mit starken Wachstumstendenzen sowohl im Sinne von beobachtbaren und beobachteten krankheitswertigen Zuständen (Indikator: Zunahme der Zahl der Diagnosen im $\mathrm{ICD}^{1}$ ), darauf spezialisierter Strukturen und der dafür gewidmeten gesellschaftlichen Ressourcen (Indikator: Anteil am BIP) sowie krankheitsbezogener Interventionen (Indikator: Zunahme der diagnostischen und therapeutischen Prozeduren).

Dafür, dass sich Ähnliches auch für gesundheitsbezogene Kommunikation/Handlungen sagen lässt (im weiteren Sinn Krankheitsprävention, Gesundheitsförderung), gibt es zunehmend klarere empirische Hinweise, die für Kickbusch eine wichtige Argumentationsbasis sind. Diese Entwicklungen sind auch schon früher in der Soziologie in verschiedener Form wahrgenommen worden - auch außerhalb der Gesundheits- und Medizinsoziologie (siehe unten). In der zweiten Auflage von Sarah Nettletons ,Sociology of Health and Illness“ (Nettleton 2006, 2) wird von einer grundsätzlichen Verschiebung der gesellschaftlichen Aufmerksamkeit von Krankheit zu Gesundheit ausgegangen, was etwa auch im Titel des Sonderhefts 46/2006 der Kölner Zeitschrift für Soziologie und Sozialpsychologie: „Soziologie der Gesundheit“ (Wendt and Wolf 2006) sichtbar wird.

\section{Kritische Perspektiven: „Medikalisierung“ und „Healthism“}

Das Wachstum der Krankenbehandlung wurde in der Soziologie zunächst als zunehmende „Medikalisierung“ vieler Lebensbereiche unserer Gesellschaft thematisiert. Diese These behauptet eine problematische Verbreiterung des medizinischen Blicks, von medizinischen Konzepten in immer weitere Bereiche der Kontrolle sozialer Abweichung und ehemals als „natürlich“ definierter Lebensereignisse bzw. in einer anderen Terminologie ausgedrückt: die Ausweitung eines professionellen, individualisierenden bzw. biologisierenden Zugangs zur Kontrolle von (abweichendem) Verhalten und eine Zunahme professioneller Macht. Zunächst in den 1970er Jahren außerhalb der Soziologie entwickelt (Thomas Szasz, Ivan Illich), wurde die These der Medikalisierung dann aufgegriffen (z. B. von Irving Kenneth Zola rund um Fragen des Umgangs mit Behinderung) und in den letzten Jahrzehnten insbesondere vom renommierten US-Soziologen Peter Conrad weiter entwickelt - so etwa in dem 2007 erschienenen Buch mit dem Titel „The Medicalization of Society: On the Transformation of Human Conditions Into Trea- 
table Disorders“ (Conrad 2007). Ein etwas anderes Verständnis ist im Kontext der Theorie funktionaler Differenzierung entstanden, angelegt in einigen kleinen Aufsätzen von Niklas Luhmann (siehe dazu auch den Beitrag von Jürgen Pelikan in diesem Heft).

Auf die nicht primär kurativ, sondern eher präventiv orientierte Kommunikation über gesundheitliche Themen schaut ein anderer Strang der Diskussion. Schon Anfang der 1980er Jahre hat Robert Crawford im kritischen Artikel „Healthism and the medicalization of everyday life“ (Crawford 1980, 10) argumentiert, dass auch der Schutz bzw. die Erhaltung von Gesundheit und Krankheitsprävention massiv an Aufmerksamkeit gewinnen und eine Bewegung von politischer Verantwortung in Richtung individueller Zuschreibung stattfindet.

Diese kritische Perspektive auf die zunehmende Ausbreitung von Gesundheitskommunikation in vielen Lebensbereichen wurde in den letzten zwanzig Jahren auch theoretisch angereichert. Hier sind insbesondere feministische Perspektivierungen und Gender Studies anzuführen, die einerseits über eine konsequente Medizinkritik (u. a. Barbara Duden) und in der Folge dann auch über die Theoretisierung von Körperlichkeit, speziell unter Bedingungen der Gen- und Informationstechnologie eine kontinuierliche theoretische Diskussion boten.

Auch in neu entstehenden Subdisziplinen wie etwa in der „Sociology of the Body“ findet eine regelmäßige implizite oder auch explizitie Auseinandersetzung mit der Bedeutung von Gesundheit/Medizin statt (z. B. Bryan Turner).

In Anschluss an Michel Foucault, der mit seinen Konzepten der Biopolitik und der Gouvernementalität bereits in den 1970er Jahren auch im deutschen Sprachraum wichtige Anstöße zu einer anderen Perspektivierung von Gesundheit und Gesellschaft bot, findet sich im angloamerikanischen Sprachraum eine Reihe von Beiträgen zu Fragen der zunehmenden Bedeutung von Gesundheit, die explizit der westlichen (Konsum- und Medien-)Kultur gewidmet sind. So setzt sich beispielsweise Deborah Lupton 1995 in „The Imperative of Health. Public Health and the Regulated Body“ mit der Verbreitung der Public-Health-Bewegung, dem Risikodiskurs und der Rolle der Massenmedien kritisch auseinander. Diese Diskussionen sind auch im deutschen Sprachraum verstärkt wahrgenommen und weitergeführt worden (Bröckling, Krasmann, Lemke 2000).

Die radikalen Umwälzungen der Gesundheitstechnologien (Humangenetik, Bioästhetik) und die Propagierung der Vorstellung der „Machbarkeit“" von Körper und Leben haben zu einer Veränderung und Auflösung der Grenzen zwischen gesund und krank hin zu einem Kontinuum von Bessern zum Verbessern geführt. Problematisiert wird insbesondere die Radikalisierung der Machbarkeit, die in enger Verbindung mit der Auflösung kollektiver Verantwortung unter ,,neoliberalen“ Bedingungen gesehen wird. Beispielhaft können hier Analysen der Schönheitschirurgie (z. B. Maasen 2008) genannt werden. Vor allem auf psychische Dimensionen dieser Verantwortungsverschiebung („Gebot des Selbstseins“) und auf die Rolle von psychologischem Expertenwissen hat auch Nikolas Rose schon seit längerem verwiesen (Rose 1989). Die zunehmende Bedeutung von Emotionen in der Gegenwartsgesellschaft, insbesonders die Fragen um stark anwachsende Depressionsdiagnosen, führen $\mathrm{zu}$ weiteren Auseinandersetzungen um neue Felder der abermaligen „Medikalisierung“ und „Regierung“"von Lebensbereichen (u. a. Ehrenberg 2004, Rose 2008). 


\section{Anwendungsorientierte Gesundheits- und Medizinsoziologie}

In der Gesundheits- und Medizinsoziologie sind diese kritischen Beschreibungen der Entwicklung der Krankenbehandlung, aber auch von Prävention und Gesundheitsförderung im gesamtgesellschaftlichen Kontext zwar prinzipiell rezipiert worden (im Sinne einer ,sociology of medicine“), haben bisher aber, insbesondere im deutschen Sprachraum, nur vergleichsweise geringe Bedeutung erlangt. Dies kann als Auswirkung einer relativ medizinnahen Institutionalisierung dieser Subdisziplin (,sociology in medicine") gesehen werden, die dazu führt, dass sich Soziologinnen und Soziologen in diesem Bereich stärker (auch) an anderen Umwelten und nicht primär an ihrer Kerndisziplin orientieren - beeinflusst durch Möglichkeiten des Zugangs zum Feld, der Forschungsfinanzierung und auch längerfristiger Karrieremöglichkeiten. Dabei ist nicht nur die Medizin bzw. die Krankenversorgung eine Quelle von Irritation bzw. Ablenkung von der Soziologie: Public Health als angewandtes Feld mit einer starken Referenz zum politischen System und vielleicht auch ein sich ausdifferenzierendes System für Gesundheitsförderung bietet neue Chancen für berufliche Tätigkeit als Expertin/Experte, auch für Forschung - aber in der Regel in einer relativ angewandten, interventionsorientierten, pragmatischen Perspektive. Diese starke Anwendungsorientierung ließ - so scheint es - zu wenig Energie für die Kommunikation mit der „Mutterdisziplin“. So wichtig wie Gesundheit/Krankheit gesamtgesellschaftlich geworden ist/sind, sollte dieses Defizit an Theorieentwicklung bzw. einer spezifisch soziologischen Perspektive als bisher zu wenig genutzte Chance gesehen werden.

\section{Die „Gesundheitsgesellschaft“" als Herausforderung für die Soziologie}

Mit dem Buch „Die Gesundheitsgesellschaft - Megatrends der Gesundheit und deren Konsequenzen für Politik und Gesellschaft" betont Ilona Kickbusch ${ }^{2}$ im Gegensatz zur vor allem kritischen Tradition auch die Chancen der Zunahme der Relevanz von Gesundheit und gesundheitsbezogener Kommunikation. Die zentralen Thesen formulierte sie auch im Rahmen eines Vortrags bei der eingangs erwähnten Tagung 2008.

Ihre Ausgangsthese besagt, dass die moderne Gesellschaft verspätet in der „dritten Gesundheitsrevolution“ angekommen sei: Die erste (gesundheitspolitische des 19.Jahrhunderts) habe primär Überleben gesichert, die zweite den Zugang zur medizinischen Versorgung und die dritte handle von der Förderung der Gesundheit angesichts zunehmender Individualisierung und Differenzierung in einer sich rapid verändernden Welt. An den Schnittstellen der vier Domänen der Gesundheit - der persönlichen und öffentlichen, der medizinischen und marktorientierten - entsteht auf neue Art und Weise eine Konstellation, welche als „Gesundheitsgesellschaft“ bezeichnet wird, wobei Dynamik und Konsequenzen bislang weder theoretisch noch praktisch klar erfasst seien.

Die Annäherung an die Gesundheitsgesellschaft und die Umbrüche, die Anfang des 21. Jahrhunderts erfolgen, umfassen nach Kickbusch drei zentrale Prozesse:

- Die De-Territorialisierung von Gesundheit: Sie ist überall.

- Gesundheit als gesellschaftliche Triebkraft: Sie ist machbar.

- Gesundheit als aktives Handeln: Jede Entscheidung ist auch eine Gesundheitsentscheidung. 
Aufgrund einer grundsätzlichen Veränderung des Gesundheitsbegriffes unterscheide sich dieser Prozess grundsätzlich von „Medikalisierung“. Beide Prozesse - die Entwicklung der Gesundheitsgesellschaft und die Medikalisierung - befänden sich in Expansion, überschneiden sich, bekämpfen sich auch, gehen aber auch Verbindungen miteinander ein.

Dies ziehe eine radikale Umorientierung und Neugestaltung von Gesundheitsangeboten und Krankheitsversorgung nach sich: Gesundheit wandere aus dem Versorgungssystem heraus in das soziale und wirtschaftliche Leben - ihr Leitsatz sei Expansion, ihre Ausdrucksform Kommunikation. Es bilde sich ein neues aktives und sehr weit gefasstes Gesundheitsverständnis heraus, spirituelle Verästelungen eingeschlossen. Gesundheit sei in allen Lebensbereichen präsent und zusehends virtuell vermittelt: „Lebensstile“ sind „Gesundheitsstile“, und Gesundheitskompetenz wird immer bedeutsamer. Der Markt und die Selbstfindung biete jeder/jedem „ihre/seine“ Gesundheit, und „die gesunde Wahl" sei nun Marketingprämisse vieler Produzenten von Konsumgütern. Wellness sei einer der am schnellsten wachsenden Wirtschaftszweige im Privatsektor, und Gesundheitsinformationen erzielen mehr Hits im Internet als Pornographie.

Diese Prozesse führten nach Kickbusch zu Paradoxien, Ambivalenzen und Ungleichheiten. Denn die Gesundheitsgesellschaft sei nicht per se „gesünder“: Sie ist von weit reichenden Ungleichheiten geprägt und von neuen Problemen überfordert (psychische Erkrankungen, Altersdemenz, virtuelle Süchte).

Derzeit gibt es - so Kickbusch-erst wenige politische Mechanismen, die eine adäquate Antwort auf diese neuen de-territorialisierten „Gesundheitslandschaften“ ermöglichen könnten. Erste Ansätze vermittelten sich jedoch in neuen Ansätzen der „Gesundheit in allen Politikbereichen“. Dabei ginge es nicht nur um neue politische Strategien, sondern auch um eine Diskussion über die Werte und ethischen Grundlagen der Gesundheitsgesellschaft. Denn schon jetzt bahne sich eine vierte Gesundheitsrevolution an, deren Fokus auf prädiktiver Medizin liege, der Machbarkeit von Gesundheit und Perfektion.

Prinzipiell kann die Frage gestellt werden, ob der Vorschlag von Kickbusch eine im engeren Sinn soziologische Theorie oder eine soziologisch-theoretisch fundierte Gegenwartsdiagnose ist oder ob es sich eher um ein politisches Buch in praktischer Absicht handelt oder ob hier der Vorschlag für eine Reflexionstheorie der Public Health oder eines neu entstehenden Funktionssystems für Gesundheitsförderung vorliegt (so Pelikan in diesem Heft). Die Herausgeber/innen laden die Leser/innen ein, sich selbst ein Bild zu machen, zunächst auf Basis der hier in der Einleitung referierten Thesen, mehrerer Publikationen (Kickbusch 2006; Kickbusch 2007; McQueen et al. 2007) bzw. der Resonanz, den der Vorschlag von Kickbusch in den Beiträgen des Heftes erzeugt hat. ${ }^{3}$

\section{Die Beiträge}

In einer Foucault'schen Denktradition steht der Beitrag von Monica Greco. In ihrem Artikel „Thinking beyond polemics: approaching the health society through Foucault" setzt sie sich mit dem Paradox der „Gesundheitsgesellschaft“ auseinander: einer Omnipräsenz von Gesundheit bei gleichzeitigem Aufkommen neuer Pathologien und weiterer 
Ungleichheiten. Sie analysiert aus einer Foucault'schen Perspektive das Dilemma von Gesundheitsförderung als radikalem Projekt und der Gesundheitsgesellschaft als „mainstream-Realität". Mit Foucaults Theorie zur Biopolitik als charakteristische Form der Machtrelationen in der Moderne, welche als Lebensmacht wirkt, wird deutlich, wie eine historische Kontextualisierung der Fragestellung und die Sicht auf den produktiven und reflexiven Aspekt von Macht und damit auch von Gesundheit helfen, den aktuellen Gesundheitsimperativ zu verstehen. Gesundheit erscheint damit heute auch als wesentlicher Bestandteil von Selbst-Regierung. Um neoliberale Rationalitäten, die alles (Leben) einem ökonomischen Blick der Effizienz unterwerfen, und die ubiquitäre Dominanz von ,Gesundheit' zusammenzulesen, zeigt Greco, wie Foucaults Konzept der Gouvernementalität für ein besseres Verständnis nutzbar gemacht werden kann. Die spät-moderne Sorge um sich selbst exemplifiziert sie am Beispiel der sog. Wellness-Revolution und der Problematik der „Wahl“, verbunden mit dem (individualisierten) Risiko, wie es gerade für die neue Gesundheitskultur symptomatisch ist. Sie betont mit Foucault: „What we get from Foucault is a warning: not to believe a discourse that reduces the meaning of our freedom to the exercise of choosing between a range of pre-defined options". Eine weitere Reflexion gilt der Genetik und der damit verbundenen Verschiebung von Grenzen zwischen Leben und Artifiziellem, welche eine neue Qualität in die Debatten um Gesundheit einführt. Schließlich setzt sich Greco mit Empowerment - einem zentralen Schlagwort der Gesundheitsförderung - auseinander, welches zugleich einen Legitimationsrahmen der Gesundheitsgesellschaft darstellt. Referierend auf Annemarie Mol könne die Frage des Empowements neu definiert werden: Nicht einfach zu fragen sei, wer ermächtigt wird, sondern vor allem auch, was damit gestärkt wird: „,questions as to what forms of empowerment we might associate with health, and claim under the name."

Jürgen Pelikan titelt seinen Beitrag „Ausdifferenzierung von spezifischen Funktionssystemen für Krankenbehandlung und Gesundheitsförderung oder: Leben wir in der ,Gesundheitsgesellschaft'?“ Der Artikel schließt an Ilona Kickbuschs Diagnose „Gesundheitsgesellschaft" an und greift Beschreibungen von sich verstärkenden krankheitsbezogenen und neuen gesundheitsbezogenen sozialen Phänomenen auf. Diese Trends werden aber nicht wie bei Kickbusch als „Gesundheitsgesellschaft“ oder bei Bauch (1996) als „gesellschaftlicher Leitcode“ interpretiert, sondern mit der „neoklassischen“ soziologischen Systemtheorie (in der Fassung Luhmanns) als weitere Ausdifferenzierung der funktionalen Differenzierung in der Moderne rekonstruiert. Dazu wird in einem ersten Schritt ein systemtheoretisch basiertes Modell von somato-psycho-sozialer positiver Gesundheit und Krankheit mit den Dimensionen persönlicher Funktionstüchtigkeit, Wohlbefinden und Attraktivität skizziert. Aufbauend auf bzw. in Abgrenzung zu bisherigen systemtheoretisch ansetzenden Versuchen wird ein Funktionssystem Krankenbehandlung/Medizin diagnostiziert und diskutiert. Es wird analysiert, welche Indizien für eine (evolutionäre) Entwicklung eines Funktionssystems für Gesundheitsförderung rund um die Steigerbarkeit positiver Gesundheit gefunden werden können bzw. wie das vermehrte Aufgreifen von gesundheitsbezogenen Themen in anderen Funktionssystemen als Referenz verstanden werden kann.

Eine dritte Theorieperspektive auf die "Gesundheitsgesellschaft" wird in der Forschungsnotiz von Regina Brunnett "Zum Mehrwert von Gesundheit im Postfordismus“ 
skizziert. Sie untersucht, welchen Sinn jene empirischen Phänomene, die Ilona Kickbusch als Indizien für die Entstehung einer „Gesundheitgesellschaft“ bezeichnet, in einem regulationstheoretischen (marxistisch-strukturalistischen) Rahmen machen können, und was das für Fragen rund um soziale Ungleichheit heißen kann. Angeschlossen wird dabei an die These, dass sich Gesundheit seit den 1980er Jahren zu einem der höchsten individuellen Werte und als treibende ökonomische, soziale und politische Kraft entwickelt hat. Auf der Grundlage einer diskursanalytischen Methodik und unter Nutzung der Unterscheidung fordistisch/post-fordistisch für das Verständnis der Entwicklung grundsätzlicher gesellschaftlicher Organisationsformen, wird in diesem Beitrag argumentiert, dass der Wert von Gesundheit gegenwärtig eine Doppelbewegung vollzieht. Auf der einen Seite habe sich seit den 1970er Jahren eine „expansive Dynamik des Werts“ von Gesundheit entwickelt, die vom subjektiven und symbolischen Mehrwert angetrieben wird. Auf der anderen Seite werde die Aneignung des Mehrwerts von Gesundheit im „Postfordismus“ zunehmend begrenzt. Im Ergebnis - so die These - werde Gesundheit dadurch in neuartiger Weise zu einem Konstituens sozialer Ungleichheit.

Brunnetts Beitrag schließt die Gruppe der expliziten Diskussionsbeiträge zur „Gesundheitsgesellschaft“ bzw. allgemeiner: zur wachsenden Bedeutung von Gesundheit/ Krankheit in unserer Gesellschaft ab. Darauf, dass die Diskussion damit nicht abgeschlossen, sondern eher erst begonnen scheint, weist unter anderem hin, dass die deutschsprachigen Sektionen Gesundheits- und Medizinsoziologie die Theorie-Diskussion im Rahmen einer gemeinsamen Konferenz 2009 in Halle/Saale auch ein Stück fortgesetzt haben.

Mit einer etwas anderen Grundhaltung setzt der Beitrag von Thomas Abel und Dominik Schori „Der Capability-Ansatz in der Gesundheitsförderung: Ansatzpunkte für eine Neuausrichtung der Ungleichheitsforschung“ an, der versucht, die theoretischen Grundlagen der Gesundheitsförderung weiter zu entwickeln. Ausgangspunkt ist der vielfältig nachgewiesene Zusammenhang zwischen sozialer und gesundheitlicher Ungleichheit. Für diese werden zu einem wesentlichen Teil gesundheitsförderliche oder -abträgliche Lebensweisen geltend gemacht, die ihrerseits mit sozialen Merkmalen assoziiert sind. Entsprechend sind viele praktisch-politische Interventionen der Gesundheitsförderung auf eine Veränderung des Handelns von Personen und Kollektiven ausgerichtet, die zur Herstellung gesundheitlichen Wohlergehens beitragen sollen. Für die Frage nach der sozialen Kontextgebundenheit dieses Handelns, das Zusammenspiel von Lebensstilen und strukturell verankerten Lebenschancen, fehlen - so die Ausgangsthese der Autoren - der Gesundheitsförderung bis heute die passenden Forschungsmodelle und empirisch belegte Wissensbestände.

Für die Weiterentwicklung der Forschungsmodelle unternehmen Abel und Schori den Versuch, den theoretischen Rahmen des Capability-Ansatzes von Amartya Sen nutzbar zu machen und mit Erkenntnissen des Bourdieu'schen Kapitalienansatzes anzureichern. Das vorgestellte Modell differenziert analytisch zwischen den materiellen und immateriellen Ressourcen eines bestimmten sozialen Kontextes, den dort prinzipiell verfügbaren Handlungsoptionen und den durch Akteurinnen und Akteure tatsächlich realisierten Handlungen; in den Mittelpunkt des Interesses rücken die jeweiligen Umwandlungsfak- 
toren bzw. -prozesse an den beiden Schnittstellen des Modells. Beobachtbar wird in diesem Rahmen also sowohl die Kontextgebundenheit von Handlungsoptionen als auch die Akteursgebundenheit von realisierten Optionen.

Eine Vielzahl von empirisch ausgerichteten soziologischen Arbeiten behandeln mittlerweile einzelne Facetten der „Gesundheitsgesellschaft“. Die beiden für dieses Heft ausgewählten empirischen Forschungsnotizen repräsentieren zwei sehr unterschiedliche Bereiche: Medizinische Forschung und Gesundheitsförderung, Interaktions- vs. Organisationsebene, qualitative vs. quantitative Methodik.

In ihrer Studie ,,Jemand kommt zu Dir und sagt bitte“: Eine empirische Studie zur Gewebespende im Krankenhauskontext" fokussiert Milena Bister häufiger werdende Entscheidungssituationen für Patientinnen und Patienten im Zuge zunehmender Verflechtung von Behandlung und Forschung. Welche soziale Situation wird hergestellt, welche kommunikative Praxis vorgenommen, um die Zustimmung von Patientinnen und Patienten für Gewebespenden zu erhalten? Wie verhält sich diese zum normativen Rahmen des Informed-consent-Gesprächs, wie es juristischen und bioethischen Standards entspricht? Ergebnis ist, dass es die sozialen Beziehungen zwischen Patientinnen/Patienten, der Organisation und deren Repräsentantinnen und Repräsentanten sind, die zur Zustimmung beitragen: Die Patientinnen/Patienten stehen medizinischer Forschung (von der sie gerade indirekt profitieren) positiv gegenüber und wollen die Organisation, in der sie behandelt werden, und die Forscher/innen, die sich mit der Bitte an sie wenden, ebenfalls unterstützen.

In der Forschungsnotiz von Robert Griebler, Wolfgang Dür und Waldemar Kremser „Schulqualität, Schulerfolg und Gesundheit. Ergebnisse aus der österreichischen „Health Behaviour in School-Aged Children“-Studie“" wird ein quantitatives Untersuchungsmodell vorgestellt, welches die Gesundheit von Schülerinnen und Schülern als vermittelnden Faktor zwischen Schulqualität und Schulerfolg postuliert. Die empirische Überprüfung auf Basis der österreichischen Daten der Studie „Health Behaviour in School-Aged Children“ (HBSC) zeigt nicht nur, dass Schulerfolg und Gesundheit gleichermaßen von der Qualität schulischer Strukturen und Prozesse abhängen und in einem wechselseitigen Bedingungsverhältnis stehen, sondern dass sich eine gute Passung für jenes Modell ergibt, das Gesundheit als Voraussetzung für Schulerfolg, d. h. als zentrale Bildungsressource annimmt. Damit wird für den Schulbereich eine neue Perspektive auf Gesundheit vorgeschlagen, wie sie in Bezug auf Wirtschaftsbetriebe bereits gut etabliert und belegt ist. Konsequenterweise schlagen die Autoren integrierte Programme des schulischen Qualitätsmanagements und der schulischen Gesundheitsförderung vor. Die Gesundheit rückt damit von einem „Nebeneffekt“" sozialer Prozesse ins Zentrum der Aufmerksamkeit und wird zugleich als Mittel zum Zweck für andere soziale Prozesse thematisiert - eine Betrachtungsweise, die ganz in Einklang mit den Thesen von Ilona Kickbusch steht.

Die Rezension von Maximilian Fochler zum Sammelband von Irmhild Saake, Werner Vogd (Hrsg.): „Moderne Mythen der Medizin. Studien zur organisierten Krankenbehandlung“ rundet das Themenheft zur „Soziologie der Gesundheitsgesellschaft ab. 
Aus unserer Perspektive stellt das vorliegende ÖZS-Schwerpunktheft einen weiterführenden Beitrag zu einer soziologisch-theoretischen Auseinandersetzung mit aktuellen Entwicklungen des gesellschaftlichen Umgangs mit Gesundheit dar. Wir hoffen, das Interesse der Leserinnen und Leser geweckt zu haben, seien sie nun selbst in die Diskussion um die „Gesundheitsgesellschaft“ involviert oder interessierte Beobachterinnen und Beobachter einer theoretisch und empirisch orientierten Medizin- und Gesundheitssoziologie.

\section{Anmerkungen}

$1 \quad$ ICD $=$ International Statistical Classification of Diseases and Related Health Problems, WHO.

2 Sozial- und Gesundheitswissenschaftlerin, Beraterin, und nicht zuletzt Leitfigur der Gesundheitsförderungs- und New Public Health Bewegung.

3 Ein ausführlicherer Artikel von llona Kickbusch war für dieses Heft zwar eingeladen, kam aus zeitlichen Gründen aber nicht zustande.

\section{Literatur}

Bauch Jost. 1996. Gesundheit als sozialer Code. Von der Vergesellschaftung des Gesundheitswesens zur Medikalisierung der Gesellschaft. Weinheim, München: Juventa.

Bröckling Ulrich, Sabine Krasmann und Thomas Lemke. 2000. Gouvernementalität der Gegenwart. Studien zur Ökonomisierung des Sozialen. Frankfurt am Main: Suhrkamp.

Conrad Peter. 2007. The Medicalization of Society: On the Transformation of Human Conditions Into Treatable Disorders. Johns Hopkins University Press.

Crawford Robert. 1980. Healthism and the Medicalization of Everyday Life. International Journal of Health Services 10 (3): 365-388.

Ehrenberg Alain. 2004. Das erschöpfte Selbst. Depression und Gesellschaft in der Gegenwart. Frankfurt am Main: Campus.

Kickbusch Ilona. 2006. Die Gesundheitsgesellschaft. Gamburg: Verlag für Gesundheitsförderung.

Kickbusch Ilona. 2007. Responding to the health society. Health Promotion International 22 (2): 89-91.

Lupton Deborah. 1995. The Imperative of Health. Public Health and the Regulated Body. London: Sage Publications.

Maasen Sabine. 2008. Bio-ästhetische Gouvernementalität - Schönheitschirurgie als Biopolitik. In: Schön normal. Manipulationen am Körper als Technologien des Selbst, hrsg. Paula-Irene Villa. Bielefeld: Transcript-Verlag.

McQueen David, Ilona Kickbusch, Louise Potvin, Jürgen M. Pelikan, Laura Balbo und Thomas Abel. 2007. Health and Modernity: The Role of Theory in Health Promotion. New York: Springer.

Nettleton Sarah. 2006. The Sociology of Health and Illness. Cambridge: Polity Press.

Rose Nicolas. 1989. Governing the soul: the shaping of the private self. London: Free Associations Books.

Rose Nicolas. 2008. Disorders without Borders? In: Emotions. A Social Science Reader, hrsg. Monica Greco und Paul Stenner. London, New York: Routledge.

Wendt Claus und Christof Wolf. 2006. Soziologie der Gesundheit. Sonderheft 46 der Kölner Zeitschrift für Soziologie und Sozialpsychologie. Wiesbaden: VS Verlag für Sozialwissenschaften. 
Karl Krajic, Dr. phil., Key Researcher am Ludwig Boltzmann Institute Health Promotion Research in Wien, Privatdozent an der Universität Wien; Sprecher der Sektion Gesundheits- und Medizinsoziologie der Österreichischen Gesellschaft für Soziologie. Aktuelle Forschungsschwerpunkte: Gesundheitsförderung in Organisationen, insbesondere der Long Term Care, Gesundheitssystemforschung. Anschrift: Ludwig Boltzmann Institute Health Promotion Research, Untere Donaustraße 47, A-1020 Wien; E-Mail: karl.krajic@lbihpr.lbg.ac.at

Rudolf Forster, ao. Univ.-Prof. am Institut für Soziologie der Universität Wien und Key Researcher am Ludwig Boltzmann Institute Health Promotion Research in Wien. Aktuelle Forschungsschwerpunkte: Nutzer- und Bürgerbeteiligung in der Gesundheitsförderung und Gesundheitsversorgung; Selbsthilfe- und Patientenorganisationen; Gesundheitsförderung in Organisationen. Adresse: Institut für Soziologie, Rooseveltplatz 2, A-1090 Wien; E-Mail: rudolf-forster@univie.ac.at

Elisabeth Mixa, Magisterium und Doktorat in Soziologie; freie Wissenschafterin und Lektorin am Institut für Soziologie der Universität Wien; Forschungsschwerpunkte: u. a. Soziologie des Körpers und der Emotionen, Gender Studies; aktuelle Publikation: Body\&Soul. Wellness: von heilsamer Lustbarkeit und Postsexualität. 2009, Bielefeld: Transcript-Verlag (erscheint im Herbst 2009). 